\title{
SOLUTIONS OF $\left(r y^{(n)}\right)^{(n)}+q y=0$ OF CLASS $\mathscr{L}_{p}[0, \infty)$
}

\section{DON HINTON}

ABSTRACT. For a certain class of ordinary differential operators $L$, this paper determines the maximum number $m$ of linearly independent solutions of class $\mathscr{L}_{p}[0, \infty)$ of $L(y)=0$. For $L(y)=$ $\left(r y^{(n)}\right)^{(n)}+q y$ and $p=2$, the principal result is that if $\int_{0}^{t}|q|^{2} d \tau=O(t)$ as $t \rightarrow \infty$, then $m \leqq n$.

We consider first the differential operators

(1) $L_{1}(y)=(-1)^{n}\left(r y^{(n)}\right)^{(n)}+q y$ and $L_{2}(z)=(-1)^{n}\left(r z^{(n)}\right)^{(n)}+\bar{q} z$,

where $r$ and $q$ are continuous, complex-valued functions on $[0, \infty)$ with $r$ positive. A result of A. Zettl [5] states that if $q$ is real and $\int_{0}^{\infty} q^{2} d t<\infty$, then the equation $L_{1}(y)=0$ has a solution which is not of class $\mathscr{L}_{2}[0, \infty)$. In this note we investigate the number of $\mathscr{L}_{p}[0, \infty)(p>1)$ solutions of (1) under a less restrictive hypothesis than that used by A. Zettl. However, we do not obtain results for the more general differential equations considered in [5].

Define $s$ by $(1 / p)+(1 / s)=1$,

and

$$
V_{1}=\left\{y \mid L_{1}(y)=0 \text { and } y \in \mathscr{L}_{p}[0, \infty)\right\} \text {, }
$$

$$
V_{2}=\left\{z \mid L_{2}(z)=0 \text { and } z \in \mathscr{L}_{p}[0, \infty)\right\} .
$$

By taking conjugates in (1), it follows that the correspondence $y \rightarrow \bar{y}$ is oneone from $V_{1}$ onto $V_{2}$; hence $\operatorname{dim} V_{1}=\operatorname{dim} V_{2}$.

THEOREM 1. If for some $K>0$,

$$
\left(\int_{0}^{t}|q|^{s} d \tau\right)^{1 / s} \leqq K t^{1-1 / s} \quad \text { for } t \geqq 1,
$$

then $\operatorname{dim} V_{1}=\operatorname{dim} V_{2} \leqq n$.

Proof. Let $y \in V_{1}$; then as in [5], by Hölder's inequality,

$$
\begin{aligned}
\mid\left(r y^{(n)}\right)^{(n-1)}(t)- & \left(r y^{(n)}\right)^{(n-1)}(1) \mid \\
& =\left|\int_{1}^{t} q y d \tau\right| \leqq\left(\int_{1}^{t}|q|^{s} d \tau\right)^{1 / s}\left(\int_{1}^{t}|y|^{p} d \tau\right)^{1 / p} .
\end{aligned}
$$

Received by the editors May 17, 1971.

AMS 1970 subject classifications. Primary 34B20, 34B25.

Key words and phrases. Limit point, limit circle, $\mathscr{L}_{p}$ solutions.

(c) American Mathematical Society 1972 
Thus (2) implies there is a number $K_{1}$, depending on $y$, such that

$$
\left|\left(r y^{(n)}\right)^{(n-1)}(t)\right| \leqq K_{1} t^{1-1 / s} \quad \text { for } t \geqq 1 .
$$

Similarly, for $z \in V_{2}$ there is a number $K_{2}$, depending on $z$, such that

$$
\left|\left(r z^{(n)}\right)^{(n-1)}(t)\right| \leqq K_{2} t^{1-1 / s} \quad \text { for } t \geqq 1 .
$$

For $L_{1}(y)=0$ and $L_{2}(z)=0$, the Lagrange identity $[y, z]^{\prime}=0$ holds where

$$
[y, z]=\sum_{i=0}^{n-1}(-1)^{i}\left[y^{(i)}\left(r \bar{z}^{(n)}\right)^{(n-1-i)}-\left(r y^{(n)}\right)^{(n-1-i)} \bar{z}^{(i)}\right] .
$$

Suppose now $\operatorname{dim} V_{1}>n$. We will show that for some $y \in V_{1}$ and $z \in V_{2}$, $[y, z]=1$.

Let $V=\left\{y \mid L_{1}(y)=0\right\}$. Define the linear transformation $S$ from $V_{2}$ into $V$ by $S(z)=w$ means

$z^{(i)}(0)=w^{(i)}(0) \quad$ and $\quad\left(r z^{(n)}\right)^{(i)}(0)=\left(r w^{(n)}\right)^{(i)}(0), \quad i=0, \cdots, n-1$.

Since solutions of linear equations are uniquely determined by their initial values, $S$ is one-one and $\operatorname{dim} S\left(V_{2}\right)=\operatorname{dim} V_{2}$. Define the linear transformation $T$ from $V_{1}$ into $V$ by $T(y)=w$ means for $i=0, \cdots, n-1$,

$$
y^{(i)}(0)=(-1)^{i}\left(r w^{(n)}\right)^{(n-1-i)}(0) \text { and }\left(r y^{(n)}\right)^{(n-1-i)}(0)=(-1)^{i+1} w^{(i)}(0) \text {. }
$$

Then $T$ is nonsingular; hence $\operatorname{dim} T\left(V_{1}\right)=\operatorname{dim} V_{1}$. Since $\operatorname{dim} V_{1}=\operatorname{dim} V_{2}>n$ and $\operatorname{dim} V=2 n$, there is a $w \neq 0, w \in T\left(V_{1}\right) \cap S\left(V_{2}\right)$. Let $y=T^{-1}(w)$ and $z=S^{-1}(w)$. Then

$$
[y, z]=\left.\sum_{i=0}^{n-1}\left[\left(r w^{(n)}\right)^{(n-1-i)}\left(r \bar{w}^{(n)}\right)^{(n-1-i)}+w^{(i)} \bar{w}^{(i)}\right]\right|_{t=0}>0 .
$$

Multiplication of (5) by an appropriate constant yields $[y, z]=1$ for some $y \in V_{1}$ and $z \in V_{2}$.

For $a>1$, it follows from $[y, z]=1$ that

$$
\begin{aligned}
\frac{t}{n+1} & {\left[1-\frac{a}{t}\right]^{n+1}=\int_{a}^{t}\left(1-\frac{\tau}{t}\right)^{n} d \tau } \\
& =\sum_{i=0}^{n-1} \int_{a}^{t}(-1)^{i}\left[y^{(i)}\left(r \bar{z}^{(n)}\right)^{(n-1-i)}-\left(r y^{(n)}\right)^{(n-1-i)} \bar{z}^{(i)}\right]\left(1-\frac{\tau}{t}\right)^{n} d \tau .
\end{aligned}
$$

An $i$-fold integration by parts gives

$$
\begin{aligned}
& \int_{a}^{t} y^{(i)}\left(r \bar{z}^{(n)}\right)^{(n-1-i)}\left(1-\frac{\tau}{t}\right)^{n} d \tau \\
& \quad=M_{i, t}+(-1)^{i} \int_{a}^{t} y \frac{d^{(i)}}{d \tau^{(i)}}\left[\left(r \bar{z}^{(n)}\right)^{(n-1-i)}\left(1-\frac{\tau}{t}\right)^{n}\right] d \tau
\end{aligned}
$$


where $M_{i, t}$ satisfies $\left|M_{i, t}\right|=O(1)$ as $t \rightarrow \infty$. From (4) we conclude that for some $N_{1}>0$ and $k=1, \cdots, n$,

$$
\left|\left(r \bar{z}^{(n)}\right)^{(n-k)}(t)\right| \leqq N_{1} t^{k-1 / s} \quad \text { for } t \geqq 1 .
$$

An application of Leibniz's rule and (8) yields an $N_{2}>0$, depending only on $z$, such that

$$
\left|\frac{d^{(i)}}{d \tau^{(i)}}\left[\left(r \bar{z}^{(n)}\right)^{(n-1-i)}\left(1-\frac{\tau}{t}\right)^{n}\right]\right| \leqq N_{2} \tau^{1-1 / s} \quad \text { for } 1 \leqq \tau \leqq t .
$$

Applying this inequality to (7), we obtain

$$
\begin{aligned}
& \left|\int_{a}^{t} y^{(i)}\left(r \bar{z}^{(n)}\right)^{(n-1-i)}\left(1-\frac{\tau}{t}\right)^{n} d \tau\right| \\
& \quad \leqq\left|M_{i, t}\right|+N_{2}\left(\int_{a}^{t}|y|^{p} d \tau\right)^{1 / p}\left(\int_{a}^{t} \tau^{s-1} d \tau\right)^{1 / s} .
\end{aligned}
$$

Since $\left(\int_{a}^{t} \tau^{s-1} d \tau\right)^{1 / s} \leqq t /(s)^{1 / s}$, we have from inequality (9) that

$$
\begin{gathered}
\limsup _{t \rightarrow \infty} \frac{1}{t} \sum_{i=0}^{n-1}\left|\int_{a}^{t} y^{(i)}\left(r \bar{z}^{(n)}\right)^{(n-1-i)}\left(1-\frac{\tau}{t}\right)^{n} d \tau\right| \\
\leqq n N_{2}\left(\int_{a}^{\infty}|y|^{p} d \tau\right)^{1 / p} /(s)^{1 / s} .
\end{gathered}
$$

Since $N_{2}$ does not depend on $a$, the right-hand side of $(10)$ is $<1 / 4(n+1)$ for sufficiently large $a$. In a similar manner we may prove that with $y$ and $z$ interchanged, the limit superior as $t \rightarrow \infty$ of $(10)$ is $<1 / 4(n+1)$. However, this gives a contradiction to (6) since

$$
\operatorname{limit}_{t \rightarrow \infty} \frac{1}{t}\left\{\frac{t}{n+1}\left[1-\frac{a}{t}\right]^{n+1}\right\}=\frac{1}{n+1} .
$$

As a corollary to Theorem 1 we obtain the deficiency indices of the formally selfadjoint operator $L$ where

$$
L(y)=\left(r y^{(n)}\right)^{(n)}+Q y .
$$

Corollary. Let $Q$ be a real continuous function on $[0, \infty)$ such that $\int_{0}^{t} Q^{2} d \tau=O(t)$ as $t \rightarrow \infty$. If $L$ is given by (11), then the equation $L(y)=\lambda y$, $\operatorname{Im} \lambda \neq 0$, has exactly $n$ linearly independent solutions of class $\mathscr{L}_{2}[0, \infty)$.

Proof. If we choose $q=Q-\lambda$, then an application of Theorem 1 gives that $L(y)=\lambda y$ has at most $n$ linearly independent solutions of class $\mathscr{L}_{2}[0, \infty)$. However, the classical theory of deficiency indices (cf. [3, Chapter V]) gives that $L(y)=\lambda y, \operatorname{Im} \lambda \neq 0$, has at least $n$ linearly independent solutions of class $\mathscr{L}_{2}[0, \infty)$ with no restrictions on the growth of $Q$. 
For $p=2$, additional results are given in [2] under which $\operatorname{dim} V_{1} \leqq n$. The conditions on $q$ in [2] are in the form of boundedness conditions on the growth of $q$ and are independent of the conditions considered here.

The arguments above may be adapted to certain odd-order equations. For example, with $r$ and $q$ as before, define

$$
L_{3}(y)=\left(r y^{\prime}\right)^{\prime \prime}+q y \quad \text { and } L_{4}(z)=\left(r z^{\prime \prime}\right)^{\prime}-\bar{q} z .
$$

Then $L_{3}(y)=0$ and $L_{4}(z)=0$ gives $[y, z]^{\prime}=0$ where

$$
[y, z]=y\left(r \bar{z}^{\prime \prime}\right)-\left(r y^{\prime}\right) \bar{z}^{\prime}+\left(r y^{\prime}\right)^{\prime} \bar{z} .
$$

If $V_{3}=\left\{y \mid L_{3}(y)=0\right.$ and $\left.y \in \mathscr{L}_{p}[0, \infty)\right\}, V_{4}=\left\{z \mid L_{4}(z)=0\right.$ and $\left.z \in \mathscr{L}_{p}[0, \infty)\right\}$, and $\operatorname{dim} V_{3}+\operatorname{dim} V_{4}>3$, we may as before show that for some $y \in V_{3}$ and $z \in V_{4},[y, z]=1$. If $q$ satisfies $\int_{0}^{t}|q|^{s} d \tau=O\left(t^{s-1}\right)$ as $t \rightarrow \infty$, then for $y \in V_{3}$ and $z \in V_{4}$ we have $\left|\left(r y^{\prime}\right)^{\prime}(t)\right|$ and $\left|\left(r z^{\prime \prime}\right)(t)\right|$ are $O\left(t^{1-1 / s}\right)$ as $t \rightarrow \infty$. From

$$
\begin{aligned}
\frac{t}{2}\left(1-\frac{a}{t}\right)^{2} & =\int_{a}^{t}\left(1-\frac{\tau}{t}\right) d \tau \\
& =\int_{a}^{t}\left\{y\left(r \bar{z}^{\prime \prime}\right)-\left(r y^{\prime}\right) \bar{z}^{\prime}+\left(r y^{\prime}\right)^{\prime} \bar{z}\right\}\left(1-\frac{\tau}{t}\right) d \tau
\end{aligned}
$$

we obtain, after integrating $\int_{a}^{t}\left(r y^{\prime}\right) \bar{z}^{\prime}(1-\tau / t) d \tau$ by parts, a contradiction as in the proof of Theorem 1 . This gives $\operatorname{dim} V_{3}+\operatorname{dim} V_{4} \leqq 3$. However, in general, $\operatorname{dim} V_{3} \neq \operatorname{dim} V_{4}$, e.g., consider $r=1, q=-1-i$.

We note also that the proof given by A. Zettl may be adapted to more general equations. For example, let

$$
L_{5}(y)=\left[\left(r y^{\prime \prime}\right)^{\prime}+p y^{\prime}\right]^{\prime}+q y
$$

where $r$ and $q$ are as before and $p$ is a continuous, complex-valued function on $[0, \infty)$. We now show that if $V_{5}=\left\{y \mid L_{5}(y)=0\right.$ and $\left.y \in \mathscr{L}_{p}[0, \infty)\right\}$, then the condition $\int_{0}^{t}|q|^{s} d \tau=O\left(t^{s-1}\right)$ as $t \rightarrow \infty$ implies that $\operatorname{dim} V_{5} \leqq 3$.

Suppose to the contrary that $\operatorname{dim} V_{5}=4$. Choose solutions $y_{1}, y_{2}, y_{3}, y_{4}$ of $L_{5}(y)=0$ so that $Y(0)=I$ where

$$
Y=\left[\begin{array}{cccc}
y_{1} & y_{2} & y_{3} & y_{4} \\
y_{1}^{\prime} & y_{2}^{\prime} & y_{3}^{\prime} & y_{4}^{\prime} \\
r y_{1}^{\prime \prime} & r y_{2}^{\prime \prime} & r y_{3}^{\prime \prime} & r y_{4}^{\prime \prime} \\
\left(r y_{1}^{\prime \prime}\right)^{\prime}+p y_{1}^{\prime} & \left(r y_{2}^{\prime \prime}\right)^{\prime}+p y_{2}^{\prime} & \left(r y_{3}^{\prime \prime}\right)^{\prime}+p y_{3}^{\prime} & \left(r y_{4}^{\prime \prime}\right)^{\prime}+p y_{4}^{\prime}
\end{array}\right] .
$$

The Wronskian $W=\operatorname{det} Y$ is constant and expanding $W$ along the last row of $Y$ we obtain

$$
1=W=\sum_{i=1}^{4} Z_{i}\left[\left(r y_{i}^{\prime \prime}\right)^{\prime}+p y_{i}^{\prime}\right]
$$


where $Z_{i}$ is the cofactor of $\left(r y_{i}^{\prime \prime}\right)^{\prime}+p y_{i}^{\prime}$. By differentiation it may be shown that $Z_{i}=(-1)^{i} y_{5-i}$. As above we may show that for each $i$,

$$
\left|\left[\left(r y_{i}^{\prime \prime}\right)^{\prime}+p y_{i}^{\prime}\right](t)\right|=O\left(t^{1-1 / s}\right) \quad \text { as } t \rightarrow \infty .
$$

From equation (12) we obtain for $a>0$,

$$
t-a=\int_{a}^{t} W d \tau \leqq \sum_{i=1}^{4}\left(\int_{a}^{t}\left|y_{5-i}\right|^{p} d \tau\right)^{1 / p}\left(\int_{a}^{t}\left|\left(r y_{i}^{\prime \prime}\right)^{\prime}+p y_{i}^{\prime}\right|^{s} d \tau\right)^{1 / s}
$$

Application of (13) in (14) now yields a contradiction as in the proof of Theorem 1.

Without additional assumptions on $r$ and $p$, it is possible to have $\operatorname{dim} V_{5}=3$. W. N. Everitt [1] mentions the example of A. D. Wood,

$$
y^{(\mathrm{iv})}+\left(t^{2} y^{\prime}\right)^{\prime}+y=\lambda y, \quad \operatorname{Im} \lambda \neq 0,
$$

which has three linearly independent solutions of class $\mathscr{L}_{2}[0, \infty)$. Results of P. Walker [4] show that the equation,

$$
\left(t^{\alpha} y^{\prime \prime}\right)^{\prime \prime}+\left(t^{\beta} y^{\prime}\right)^{\prime} \pm t^{\gamma} y=\lambda y, \quad \operatorname{Im} \lambda \neq 0,
$$

has three linearly independent solutions of class $\mathscr{L}_{2}[1, \infty)$ if $\alpha<4, \beta>2$, and $\gamma \leqq 0$.

\section{REFERENCES}

1. W. N. Everitt, Some positive definite differential operators, J. London Math. Soc. 43 (1968), 465-473. MR 37 \#3086.

2. D. Hinton, Limit point criteria for differential equations, Canad. J. Math. (submitted).

3. M. A. Naĭmark, Linear differential operators. Part II: Linear differential operators in Hilbert space, GITTL, Moscow, 1954; English transl. of 1st ed., Ungar, New York, 1967. MR 16, 702.

4. P. Walker, Deficiency indices of fourth-order singular differential operators, J. Differential Equations 9 (1971), 133-140.

5. A. Zettl, A note on square integrable solutions of linear differential equations, Proc. Amer. Math. Soc. 21 (1969), 671-672.

Department of Mathematics, University of Tennessee, Knoxville, Tennessee 37916 